\title{
Distributed Adaptive Learning Framework for Wide Area Monitoring of Power Systems Integrated with Distributed Generations
}

\author{
Kang $\mathrm{Li}^{1,2}$, Yuanjun Guo ${ }^{1}$, David Laverty ${ }^{1}$, Haibo $\mathrm{He}^{3}$, Minrui $\mathrm{Fe}^{2,4}$ \\ ${ }^{1}$ School of Electronics, Electrical Engineering and Computer Science, Queens University Belfast, Belfast, UK \\ ${ }^{2}$ UK-China Science Bridge Joint Laboratory on Energy and Automation, Shanghai University, \\ China and Queens University Belfast, UK \\ ${ }^{3}$ Department of Electrical, Computer, and Biomedical Engineering University of Rhode Island, USA \\ ${ }^{4}$ School of Mechatronics and Automation Shanghai Uni-versity, China \\ Email: k.li,yguo01@qub.ac.uk, david.laverty@qub.ac.uk, he@ele.uri.edu,mrfei@staff.shu.edu.cn
}

Received April, 2013

\begin{abstract}
This paper presents a preliminary study of developing a novel distributed adaptive real-time learning framework for wide area monitoring of power systems integrated with distributed generations using synchrophasor technology. The framework comprises distributed agents (synchrophasors) for autonomous local condition monitoring and fault detection, and a central unit for generating global view for situation awareness and decision making. Key technologies that can be integrated into this hierarchical distributed learning scheme are discussed to enable real-time information extraction and knowledge discovery for decision making, without explicitly accumulating and storing all raw data by the central unit. Based on this, the configuration of a wide area monitoring system of power systems using synchrophasor technology, and the functionalities for locally installed open-phasor-measurement-units (OpenPMUs) and a central unit are presented. Initial results on anti-islanding protection using the proposed approach are given to illustrate the effectiveness.
\end{abstract}

Keywords: Smart Grid; Anti-islanding Protection; Distributed Agents; Incremental Learning; Monitoring and Control

\section{Introduction}

We first consider a generic scenario where a distributed adaptive real-time learning system is often placed within a data intensive environment and actively interacts with it [1-3]. The application environment may be structured such as monitoring and control of large-scale power systems or unstructured where the objects to be monitored and operated are dynamic and uncertain, such as monitoring and contingency control of large scale renewable generations [4]. Figure 1 illustrates such a generic scenario for smart grid monitoring, operation and control.

For such applications, a large amount of spatial-temporal and often heterogeneous data is acquired and processed through a distributed network of static and mobile data acquisition nodes/agents. A learning system is put in place to adaptively learn and accumulate meaningful information and knowledge to generate a global model for situational awareness and to make decisions to be implemented by a distributed network of actuators. Each node in the distributed sensor and actuator networks possesses some computation power to either pre-process local and temporal data or to locally implement the decisions from the core or central unit through task decomposition and coordination among the nodes. In addition to communication with the core, the nodes may communicate with each other or a node may communicate with other nodes of close proximity.

Among various issues to be addressed for such complex systems, we mainly consider the data fusion to support overall system operation and control. The core aim is to build a dynamic, global, and abstract model, based on which consequences are inferred and decisions are made. However, the major challenge is that data obtained

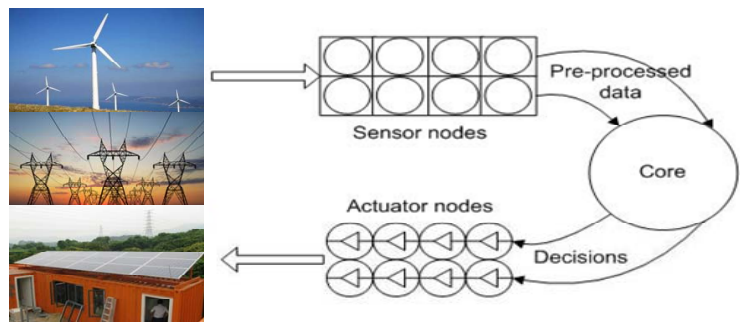

Figure 1. A generic scenario in smart grid applications. 
from such systems are often of high dimension, imbalanced, multi-modal, multi timescale, spatial-temporal, heterogonous, unsynchronized and uncertain nature, coupled with large interdependencies and interactions between human and machine systems. It presents a major challenge to effectively and efficiently collect, transmit, store, monitor, process, integrate and analyze these data for information integration and accumulation, extraction, knowledge discovery, situation awareness, and decision making in operation and control of the whole systems. In particular, we consider the following challenges [3,5-7]:

1) Dimension of time and multi-modality. In a complex system, time has a multidimensional characteristic as different types of events involve the assembly of many finer events with each having different time scale. Further, the measurement for even a single event may involve multiple modes using different sensors.

2) Discreteness. Complex systems often exhibit discrete behavior. Also, microscopic granularity will help with the emergence of macroscopic objects in both space and time dimensions. It is therefore important to embed such a feature in the system model.

3) Connections and dynamic networks. The emergence of overall behavior of a complex system depends on the dynamic and often nonlinear interaction among all its parts and elements. There is a need for a network theory and framework to reflect these connections over various time and space horizons.

4) Too many or too few data. Most methods for situation awareness are data-driven approaches. However, on one hand, due to the many variables and interactions, it is difficult to obtain sufficient data to build accurate model. For example, suppose a system has a moderate of 10 dimensions with each dimension falling into an interval of $[0,1]$. By first uniformly dividing each interval into ten subintervals, the whole domain is equally divided into $10^{20}$ small cubes. Assume that the function value at a fixed point in each cube is to be estimated and ten measurements in each cube are adequate to get reliable estimates. Then the total number of measurements on demand is up to $10^{21}$, which is impossible to obtain in practice. This is the well-known curse of dimensionality issue. This situation is even worse for fault diagnosis where faulty situations are often rare, while large portion of data are normal data. On the other hand, many real-world applications involve millions or billions of data records, for example, in power system monitoring, the sampling rate of portable devices for detecting the pulse discharge in electricity facilities is often at $20 \mathrm{MHz}$, which means even in one second, tens of millions of records are logged and need to be processed, and many off-the-shelf techniques for data-driven modeling become computationally impractical.

To deal with these challenges, this paper proposes to use an incremental learning framework originally proposed in [1] to facilitate real-time learning and knowledge acquisition. In particular, the details to implement a wide area power monitoring system using synchrophasor technology will be discussed, and initial results will be presented.

\section{The Incremental Learning Framework}

For real-time learning and knowledge acquisition for situation awareness in large-scale applications, He et al [1-3] proposed an incremental learning scheme, as illustrated in Figure 2. The proposed framework has three layers of organization and three directions of data flow. In the following, details will be discussed on how to implement such a framework.

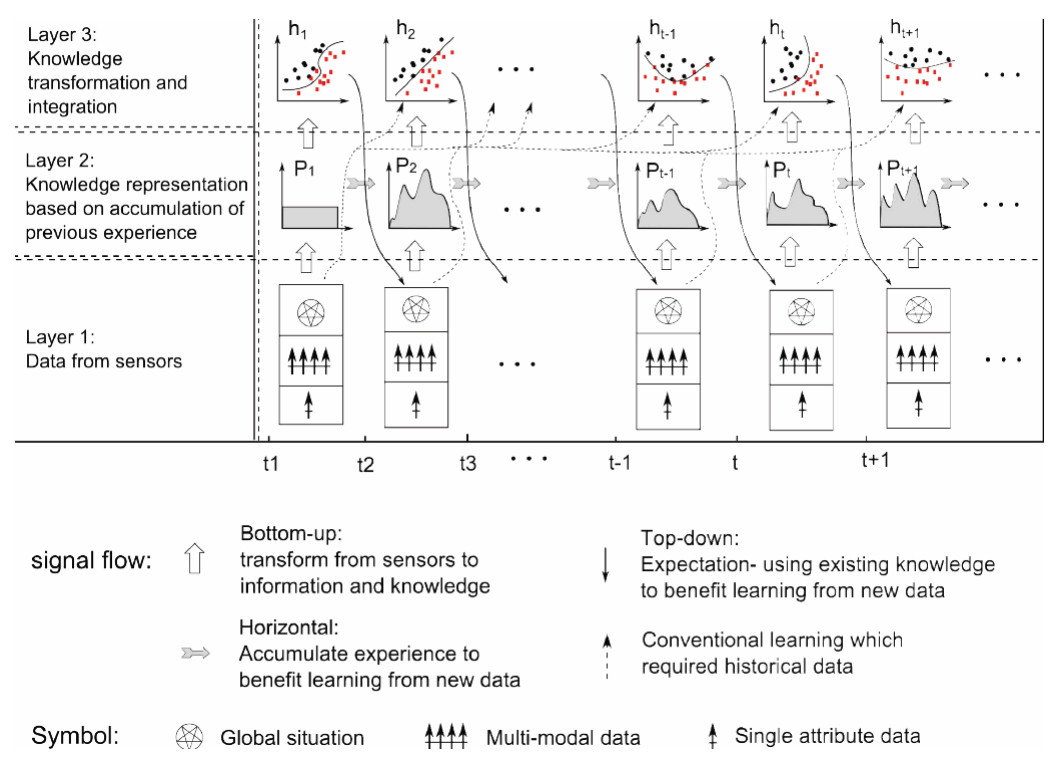

Figure 2. Incremental learning framework [1-3]. 
Layer 1: Global situation generation using streams of processed data transmitted from the distributed sensing network with locally featured information.

This layer integrates all data streams from the distributed sensing network and generates a global view of the situation using data fusion techniques, such as grid based models, distributed probability network, and Bayesian filtering technologies, etc. to deal with the integration of spatial-temporal multi-modal data. It should be noted that the streams of data transmitted from different sensors or local agents are often processed data by local sensor nodes which often have some limited computation capacity.

To avoid heavy transmission traffic (often through wireless and wired networks) and also to deal with data imbalance, the data transmitted to the second layer are often down sampled with larger time scale or are only small segment of data of interest, while local computing facilities (e.g. embedded systems) perform real-time initial data processing using e.g. filters or de-noising techniques, and data analysis using e.g. fast classification and fault diagnosis techniques to extract useful data segments or features. This helps to reduce the amount of data transmitted to the upper layer and thus enables more effective and efficient data fusion. Further, in this layer, particular attentions should also be paid to the consistence and completeness of the model to represent the global situation.

Layer 2: Knowledge extraction and representation based on the accumulation of previous experience.

This layer extracts patterns of attributes from the global view generated from layer 1 and builds a global knowledge base from the previous experience. The knowledge representation in this layer depends on the specific application, and they can be in the form of distribution functions, fuzzy rules, statistic or deterministic global models of the occurrence of certain situations or faults, or other forms that can effectively represent high-level of knowledge accumulated from previous experience.

Layer 3: Multiple hypotheses development by effective weight adjustments in the incremental learning life. This layered learning architecture enables consideration of all previous domain data and accumulates knowledge from layer 2 to any future time instance without explicit access to previously observed raw data.

In the following, details will be presented on how to implement the scheme in building a wide-area monitoring system for smart grid integrated with distributed generators, often from renewables, using synchrophasor technologies.

\section{Automatic Anti-islanding Detection Using Synchrophasors}

To address the twin challenges of tackling climate change and maintaining energy security, many countries are committed to decarbonize their energy systems. As a result, the renewable energy solution becomes an increasingly attractive and important topic, and it contributes to the sustainable development of the society. The development of hybrid power systems incorporating renewable sources represents a big step towards distributed generation (DG) and smart grid [8]. Distributed generation concerns the generation and interconnection of electricity from many small-scale and geographically distributed energy sources to the main power utility. The energy sources include renewables such as wind, tidal and wave, solar PV, and bio-fuels, etc, but also traditional ones like diesel generators. Today, some networks frequently operate with greater than $10 \%$ of their power supplied by distributed generation, and Ireland has at times operated with over $40 \%$ of generation supplied by wind energy [9]. High penetration of distributed generations has placed considerable impact on the power system planning, scheduling, operation, control, protection, and maintenance. Of particular interest here is the islanding situation where a distributed generator is supplying power to a location without power from the utility being present.

Islanding can occur intentionally, but also unintentionally, for the latter case, this is often caused by an unexpected interruption to the utility supply in a region where an embedded generator is operating in parallel to the utility grid. Islanding may pose a risk of damaging utility plant and customer connected equipment, and it also presents a danger to utility personnel working to restore the utility supply and to the public as the utility is no longer in control of power quality and earthing. For these reasons, islanded operation while connected to the utility network is generally forbidden. Methods of preventing islanding are known as anti-islanding detection, and it has become the most challenging and important aspect in designing the electrical power system with cogenerations.

An important technique in anti-islanding detection is to reliably detect the power islanding condition, and the two most common methods are Rate-of-Change -ofFrequency (ROCOF) and Vector Shift which rely on a power imbalance to detect islanding $[8,10,11]$. These techniques can fail when the power imbalance may not be large enough to activate the protection to trip the inter-tie break to disconnect the power island from the utility, or an event may occur across the grid which is however misjudged by ROCOF or Vector Shift relay as an islanding event, leading to the nuisance tripping which can further cause cascade tripping, causing significant economic and social loss. Therefore, to improve the reliable islanding condition detection has become an important research topic. 


\subsection{Introduction to Automatic Anti-islanding Detection Using Synchrophasors}

Wide-area Synchrophasor technology provides the antiislanding detector at distributed generators with real-time information, delivered using secure Internet Protocol technologies, so that nuisance trips may be avoided, sensitivity is improved and cascade tripping can be prevented [10-12].

In general, the pure sinusoidal waveform is commonly represented as a phasor $Y=Y_{r}+Y_{i}=\left(A_{m} / \sqrt{2}\right)\left(e^{j \varphi}\right)$. According to IEEE Standard C37.118.1-2011, this basic concept is adapted as the representation of AC power system sinusoidal signals. In wide-area Synchrophasor technology, PMU phasor measurements are required to be synchronized to UTC time with sufficient accuracy.

Laverty et al introduced synchrophasor for anti-islanding detection [11], and a prototype of the detector has been tested. In the method, the thresholds the detector should operate were dependent on analysis of the network, and a system of phase monitoring stations has been installed to acquire this data.

To achieve this, a reference signal is acquired from a dependable utility site, for example a transmission substation, a power station or a major node on the network that would be considered to have a utility connection at all times excluding extremely unusual circumstances. This reference signal is acquired using Phasor Measurement Unit (PMU) technology, meaning that the signal will contain at minimum the voltage amplitude, frequency and phase angle at a specific and accurate time. The standard for representing Synchrophasors is IEEE C37.118 [11,12]. The reference signal is transmitted to local distributed generators around the power system, by means of Internet Protocol (IP) telecommunications. This may be via the Internet (public network) or via a secure utility network. Internet Protocol is the preferred communications protocol due to its flexibility and ease of reconfiguration which become important should the generator be required to use a different reference site if the first becomes unavailable due to a fault or maintenance. The telecoms can be secured using IPSec tunneling, which is an integral part of the IPv6 standard.

At the generator site, the reference signal is compared to the Synchrophasor acquired at the generator terminals. The time signatures of the Synchrophasors are aligned so that phasors taken at the same instant are compared. The phase difference between the generator and the reference site are compared against known typical operating conditions. When the phase between the generator and the reference site becomes abnormally large, the generator is considered to be islanded and will be disconnected from the utility.

In principle, if at the moment islanding occurs the power imbalance between the generator and the islanded load is low than certain threshold, the generator may continue operating at the same frequency as when grid connected. However, natural variations in the grid frequency will be enough to lead to a phase difference between the generator and the utility and allow islanding to be detected.

If the power imbalance is large, the frequency of the generator will be different to that of the utility and islanding will be detected either by the frequency discrepancy, or by the rapid swing of the generators phase angle. Therefore, synchrophasor anti-islanding detector can operate in two modes. Firstly, using the phase difference, the detector will eventually detect that islanding has occurred. After approximately 2 seconds, the generator is out-of-sync with the utility supply and continuing to drift. Depending on how wide the threshold for phase difference is set, the time delay may prove excessive. Alternatively, the detector can use the rate of change of phase, or the slip frequency of the generator. This is the rate at which the generators phase slips against the utility with respect to time.

\subsection{Real-time Anti-islanding Detection using Synchrophasors}

While wide-area system monitoring using Synchrophasor offers improved opportunities for automatic detection of islanding conditions, the challenges include 1) a large amount of data are collected from each node and majority are normal data. Continuously transmitting large amount of data from a number of local sensing nodes to the core (host server or central control unit) will not help with real-time situation awareness and power system operation and control. 2) Current anti-islanding detection technologies such as Rate-of-Change-of-Frequency (ROCOF), Vector Shift, as well as phase and frequency analysis using multiple reference signals all need careful network and operation condition analysis to set appropriate thresholds. The difficulty is that the operation conditions vary with time, detection based on current single window of power system conditions may fail either to detect the islanding condition or to cause nuisance tripping. Therefore, the aforementioned incremental learning framework provides a great potential in improving the near real-time monitoring and detection performance.

To implement the proposed framework on wide-area monitoring system using Synchrophasor technologies, the distributed PMUs and the central unit are designed with different functionalities.

1) PMUs at distributed sites

PMUs used in this monitoring system are based on the OpenPMUs developed at Queen's University Belfast [11]. The OpenPMU can sample 6 channels (3 voltages, 3 currents) at $6.4 \mathrm{kHz}$, which gives 128 samples per cycle at $50 \mathrm{~Hz}$ or $60 \mathrm{~Hz}$ frequency. The ADC starts acquisi- 
tion on the rising edge of the external hardware sampling trigger whose signal is a $50 \mathrm{~Hz} / 60 \mathrm{~Hz}$ square wave which by means of a GPS receiver oscillates in phase with Coordinated Universal Time (UTC). The GPS receiver used is a Garmin GPS-18x which outputs a TTL level one-pulse-per-second (1PPS) signal, a $1 \mathrm{~Hz}$ square wave disciplined and in phase with the transition of the UTC second. Firmware on a PIC microcontroller operated as a fuzzy logic phase-locked-loop (PLL) which multiplies the 1PPS by the Synchrophasor reporting rates recommended in IEEE C37.118.1. The recommended reporting rates are 10/25/50 reports per second in a $50 \mathrm{~Hz}$ system, and 10/12/20/30/60 reports per second in a $60 \mathrm{~Hz}$ system. The PIC microcontroller also provides a time transfer mechanism to the Labview environment so that Synchrophasors can be time coded with UTC derived time.

At present, synchrophasors in OpenPMU are computed using standard Windows PC from the analog waveform data within the Labview environment. The spectrum of nominally 4-cycles at line frequency is found using FFT. The three dominant frequency bands of the spectrum are used in an iterative curve fitting algorithm. The frequency, phase and amplitude of the measured waveform are determined from the parameters of the synthesized waveform generated during curve fitting. Then, estimated Synchrophasor parameters are exported from Labview in CSV format, which contains the time code, amplitude, frequency and phase angle in ASCII strings representing decimal numbers. The CSV string is transmitted by Labview by means of UDP/IP (User Datagram Protocol over Internet Protocol) to a destination IP address and port number.

To support the incremental learning based on the afore-mentioned method, additional functionalities are needed to integrate into the existing OpenPMU: a) The PMUs as local agents should be able to communicate with other PMUs, such as for reference signals from a utility dependent site of close proximity; b) Additional pre-processing step is added, including filtering and outliers removal. c) Fast anomalies detection using ROCOF, Vector Shift, linear PCA, and dynamic PCA, etc. Once anomalies are detected, the corresponding data segment will be transmitted from the PMU to the core (central unit). If no abnormal situation is detected, then the data transmitted to the core will be further down-sampled significantly to reduce the overall data transmission load.

2) Central unit. At present, the central unit (server) at Queen's University is only used for data storage for post-event analysis. To implement the proposed incremental learning scheme, it is used as the core of the monitoring system. Basically, the central unit in the system collects all data transmitted from the PMUs, and builds an overall system view using distributed temporal-spatial model. It also uses advanced fault detection methods to monitor the whole system operation, such as PCA techniques (linear, dynamic, kernel based, nonlinear), model based, and support vector machine (SVM), etc. In this paper, we use linear PCA to detect islanding situations. Further, various islanding modes and conditions are recorded, accumulated, and used to build a fuzzy system for fast inference. Thus, knowledge and experience are accumulated, and the incremental learning framework is implemented.

Given the above design guidelines, a schematic of the wide-area system monitoring network using Synchrophasor is shown in Figure 3. The original network of PMUs has already been deployed across the Great Britain and Ireland electrical networks. This includes four PMUs in southern England, one in Manchester, one in Tealing, five on the Orkney islands, and two in Shetland. Additional PMUs operate on the Irish network providing similar data.

Data is transmitted to a central unit (server) in the host organization QUB in an ASCII plain text format and stored and for further processing and analysis, such as for post analysis of events, determination of suitable antiislanding relay event thresholds and online simulation of anti-islanding relays.

\subsection{Preliminary Results}

Three PMUs (PMU-1, PMU-4, PMU-5) in the network captured one data set containing a frequency dip caused by a $1 \mathrm{GW}$ loss in bulk generation as well as an islanding event occurred in response to the frequency dip. In the following, we show how the proposed framework is implemented.

Figures 4, 5 and 6 show that three PMUs (PMU-1, PMU-4, PMU-5) at three different sites simultaneously

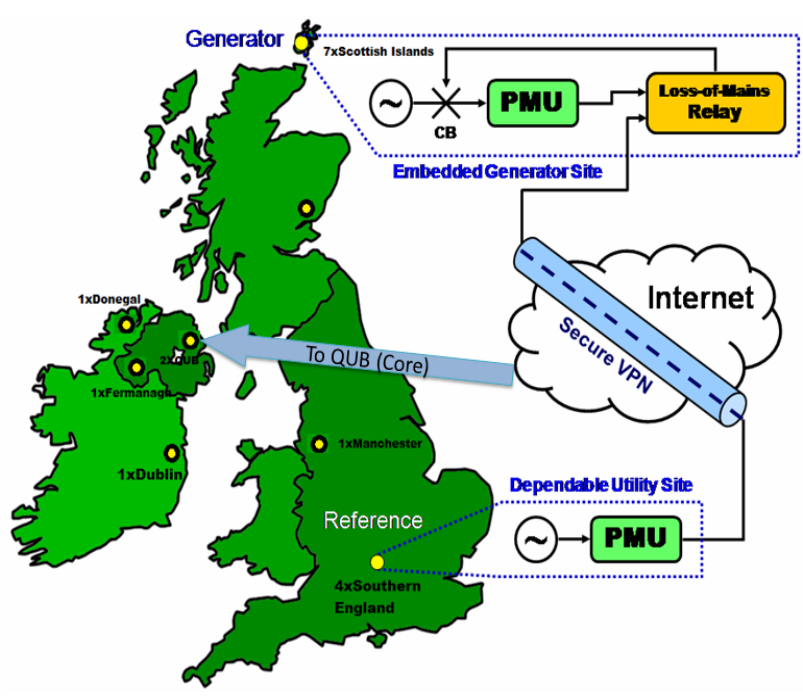

Figure 3. Synchrophasor Anti-Islanding detection scheme. 


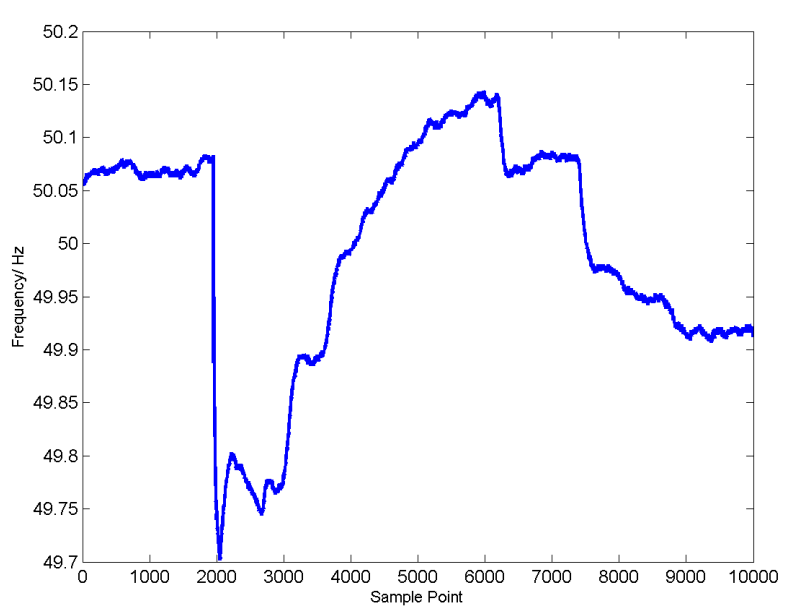

Figure 4. Frequency dip captured by PMU-1.

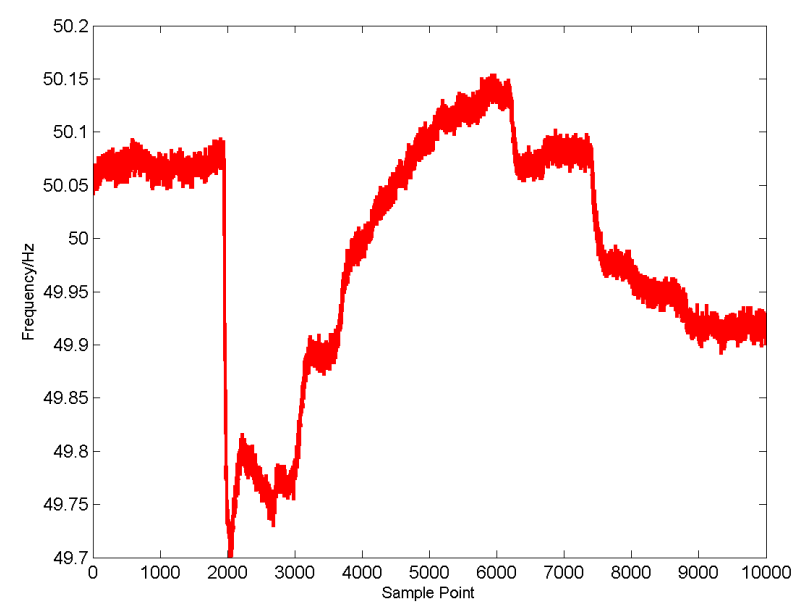

Figure 5. Frequency dip captured by PMU-4.

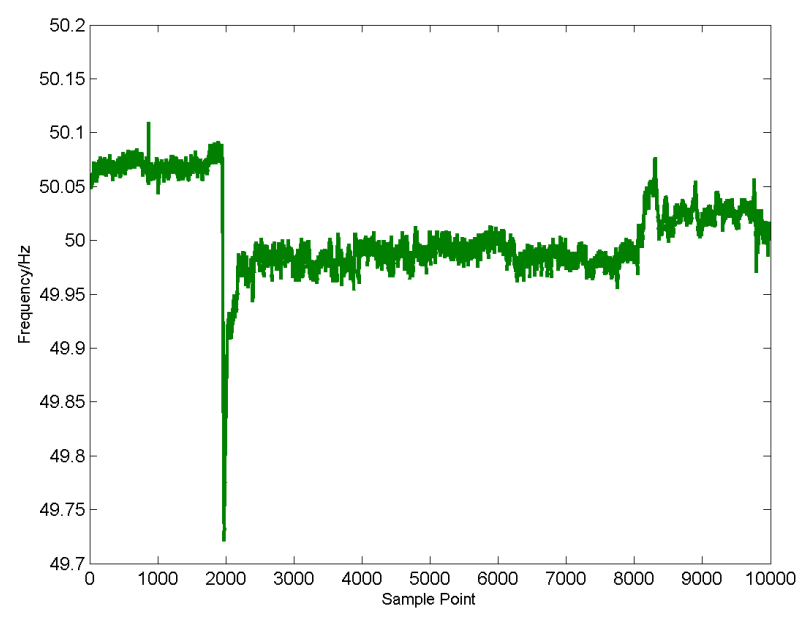

Figure 6. Frequency dip captured by PMU-5.

captured abnormal dip in frequency during a day. Figures 7, 8 and 9 show the ROCOF results, where the frequency dip lasted for 5 seconds. The detection of frequency dips using ROCOF in local PMUs can be fast

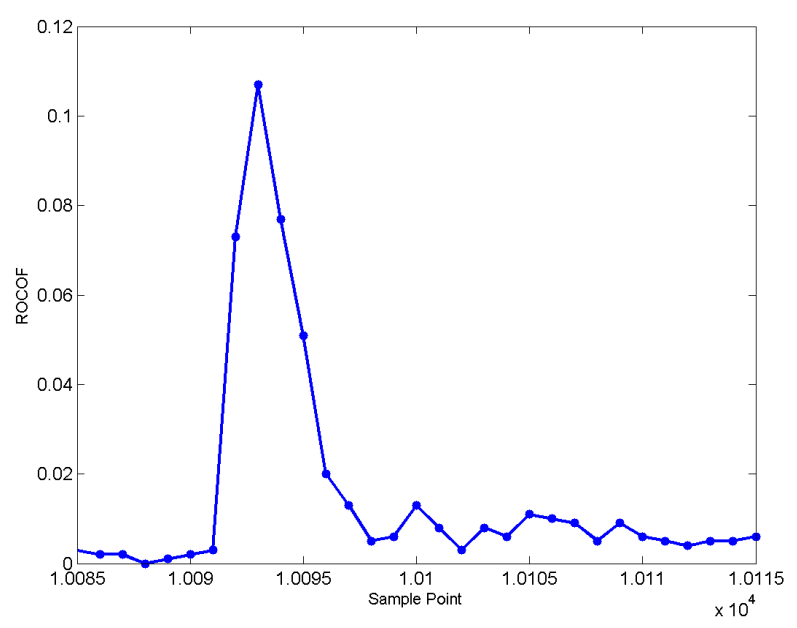

Figure 7. ROCOF results from PMU-1.

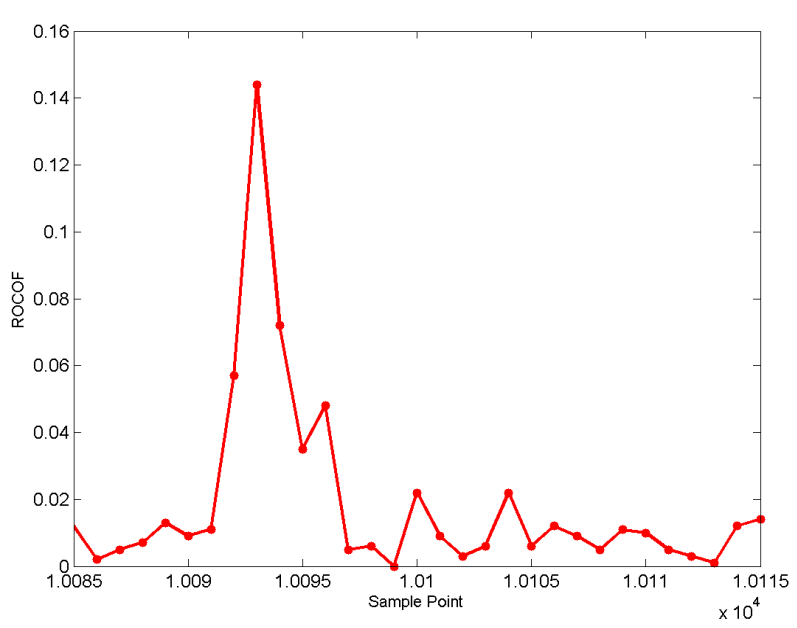

Figure 8. ROCOF results from PMU-4.

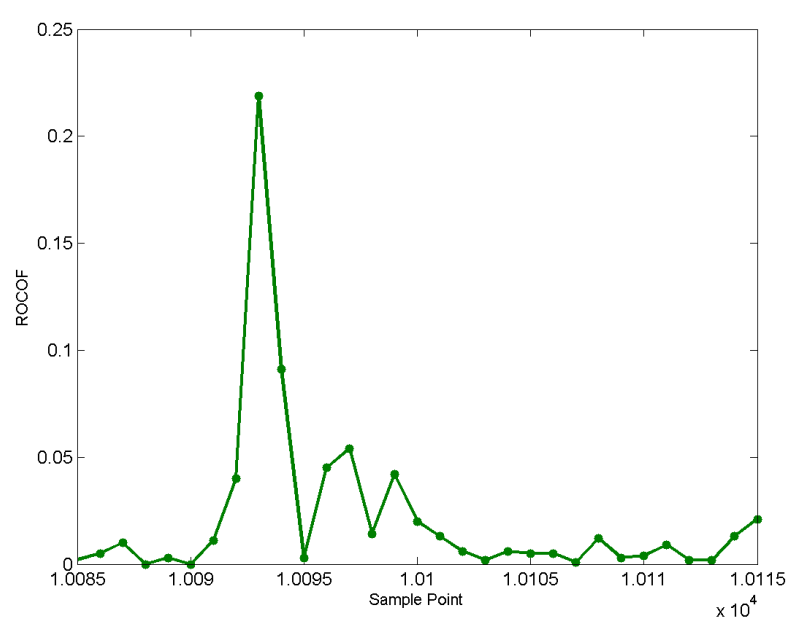

Figure 9. ROCOF results from PMU-5.

enough. In our lab simulation, it only used around $2.3 \mathrm{~ms}$ to process 5 samples, with a Dell PC with Intel(R) Core(TM) i5-2400 CPU @ 3.10 GHz, RAM 4.00 GB and 
with a 32-bit Operating system.

Once the PMUs detect the significant change of frequency, the relevant data segments are then transmitted to the central unit from these PMUs, and PCA [13][14] is used by the central unit to perform statistic analysis on the islanding situation. Figure 10 shows the detection results when PCA is used in the central unit, where particular high values indicating possible islanding event occurred.

To further distinguish the potential islanding site, the PCA based fault reconstruction method [14] is performed in the central unit, and Figure 11 shows the fault reconstruction results. While these lines in Figure $\mathbf{1 1}$ are straight but the two sections of fluctuations on each of them represent the response behavior after the frequency dip occurs. The green line of PMU-4 is different from PMU-1 and PMU-5 and with a relative high magnitude.
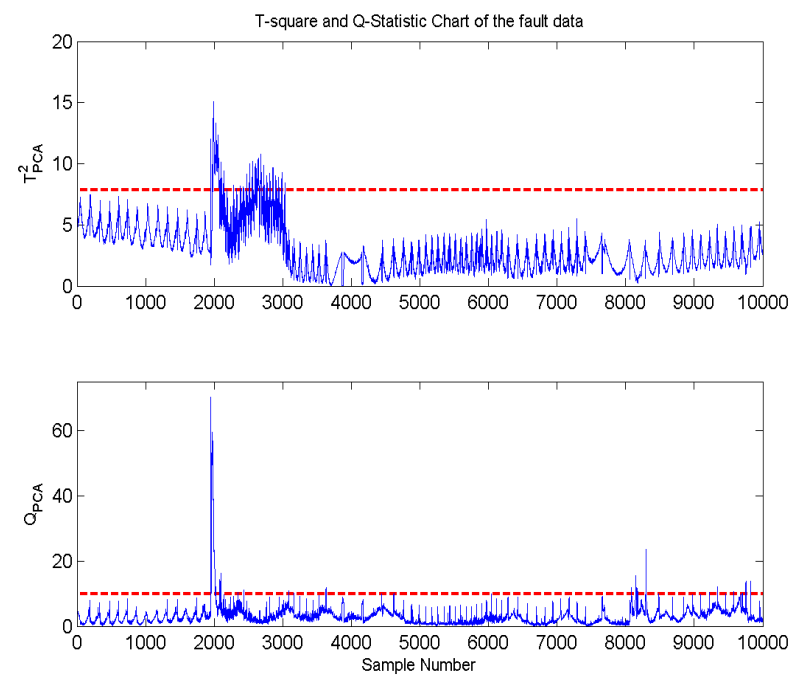

Figure 10. PCA analysis results.

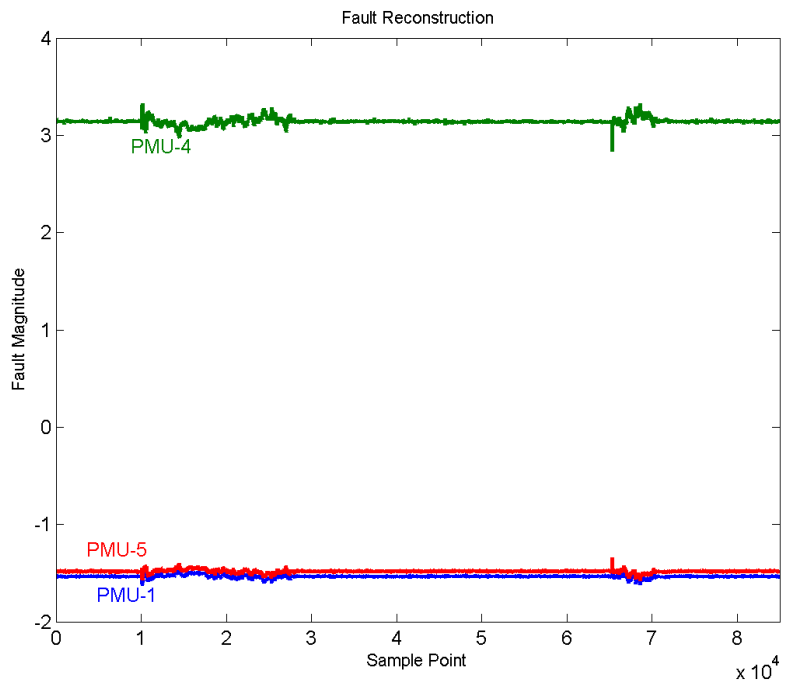

Figure 11. Fault reconstruction using PCA model.
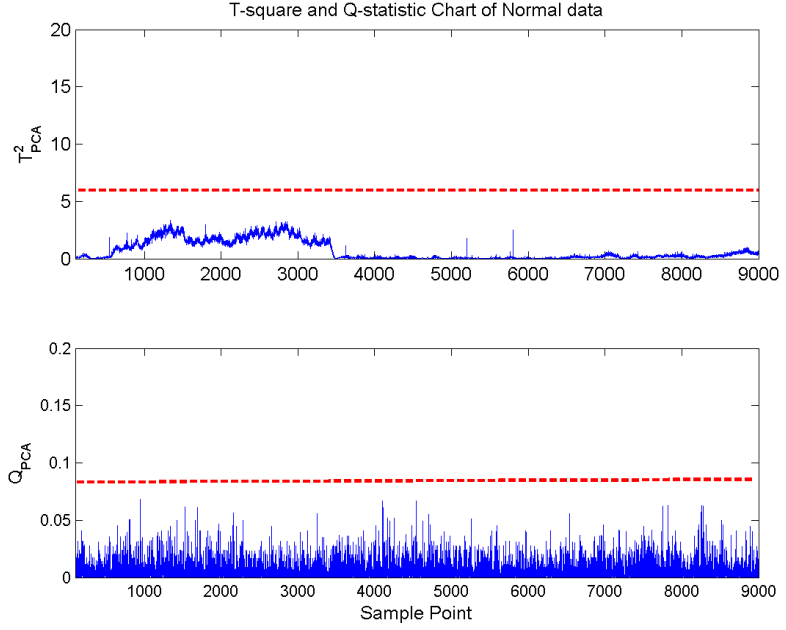

Figure 12. Normal operation conditions.

The frequency plots also show that PMU-4 was unsynchronized with the entire grid after the two big frequency changes, and PMU-4 signal kept generating power on its own frequency for a while before resynchronization. From previously accumulated experience, this case indicates the islanding occurs on the site where PMU-4 is installed. In terms of the processing time, the same PC only used $73.5 \mathrm{~ms}$ to detect the islanding situation. Finally, Figure 12 shows the PCA analysis of the central unit under normal operation conditions, which illustrates that the system can reduce false alarms if no fault event occurred.

In summary, this preliminary study has shown that the proposed wide-area power system monitoring scheme using synchrophasor technology can offer great potential in fast capturing of islanding events while reducing the number of false alarms and minimizing the amount of data transmissions from distributed synchrophasors to the central processing unit.

\section{Conclusions}

This paper has discussed the application and extension of a recently proposed novel distributed adaptive real-time learning framework for supporting wide area monitoring of distributed power generation for anti-islanding protection. The framework comprises distributed agents (OpenPMUs) for autonomous local operation, and incremental learning is used to generate global dynamic view. Initial results show that the proposed approach can correctly identify islanding events, with the benefit of significantly reduced amount of data transmitted through the network, making use of the available though limited computing capabilities in local agents. This has enabled the central unit to focus on generating a global view for situation awareness and a high-level knowledge database can be established for real-time system operation and control. It 
should be noted that this paper only presents an early stage of the development of wide-area power monitoring system using the proposed distributed incremental learning scheme, and future work will involve the development of a comprehensive suite of technologies for each PMU and for the central unit for different monitoring scenarios, and assessment of the long-term benefit of such scheme in real-time power system monitoring and control.

\section{Acknowledgements}

This work was partially supported by Engineering and Physical Sciences Research Council (EPSRC) under grants EP/F021070/1, the Key Project of Science and Technology Commission of Shanghai Municipality under Grant No.11ZR1413100, National Natural Science Foundation of China under Grants 61273040 and Shanghai Rising-Star Program (12QA1401100).

\section{REFERENCES}

[1] H. He, "Self-adaptive Systems for Machine Intelligence," Wiley-Interscience, 2011. doi:10.1002/9781118025604

[2] H. He, S. Chen, K. Li and X. Xu, "Incremental Learning From Stream Data," IEEE Trans. Neural Networks and Learning Systems, Vol. 22, No. 12, 2012, pp. 1901-1914.

[3] H. He and G. Garcia, "Learning from Imbalanced Data," IEEE Transactions Knowledge and Data Engineering, Vol. 21, No. 9, 2009, pp. 1263-1284. doi:10.1109/TKDE.2008.239

[4] F. Lafarge and J. Descombes and X. Zerubia, “Textural Kernel for SVM Classification in Remote Sensing: Application to Forest Fire Detection and Urban Area Extraction," IEEE International Conference on Image Processing, Vol. 3, 2005, p. 1096.

[5] S. Solomon and E. Shir, "Complexity, a Science at 30,"
Europhysics News, Vol. 34, 2003, pp. 54-57. doi:10.1051/epn:2003204

[6] N. Shnerb, “The Importance of Being Discrete: Life Always Wins on the Surface," Proc. Natl. Acad. Sci., Vol. 97, 2000, pp. 10 322-10 324.

[7] E. Matson, S. A. DeLoach and R. Bhatnagar, "Evaluation of Properties in Transition of Capability Based Agent Organization,” Web Intelligence and Agent Systems, Vol. 7, 2009, pp. 1-21,.

[8] N. Jenkins, R. Allan, P. Crossley, D. Kirschen and G. Strbac, "Embedded Generation," Power and Energy Series 31. London, UK: Inst. Elect. Eng, 2000. doi:10.1049/PBPO031E

[9] Eirgrid, "System Demand Data,” www.eirgrid.com, 2011.

[10] J. Jiang, J. Yang, Y. Lin, C. Liu and J. Ma, “An Adaptive PMU Based Fault Detection/location Technique for Transmission Lines. i. Theory and Algorithms," Power Delivery, IEEE Transactions on, Vol. 15, No. 2, 2000, pp. 486-493. doi:10.1109/61.852973

[11] D. Laverty, D. Morrow, R. Best and M. Cregan, “Anti-islanding Detection Using Synchrophasors and Internet Protocol Telecommunications," in Innovative Smart Grid Technologies (ISGT Europe), 2011 2nd IEEE PES International Conference and Exhibition on. IEEE, 2011, pp. 1-5.

[12] IEEE Standard for Synchrophasors for Power Systems, IEEE Std. C37.118-2005 (Revision of IEEE Std. 1344-1995).

[13] J. V. Kresta, J. F. Macgregor and T. E. Marlin. "Multivariate Statistical Monitoring of Process Operating Performance,” The Canadian Journal of Chemical Engineering, Vol. 69, No. 1, 1991, pp. 35-47.

doi:10.1002/cjce.5450690105

[14] R. Dunia and S. Joe Qin, "Subspace Approach to MultiDimensional Fault Identification and Reconstruction," AIChE Journal, Vol. 44, No. 8, 2004, pp. 1813-1831. doi:10.1002/aic.690440812 